\title{
Pediatricians and the health of future generations: Joint responsibilities and a common challenge
}

The first years of life have always been recognized in the field of pediatrics as a "critical period", envisaging the importance of a child's health since the time of conception. During this critical period, environmental factors trigger a process called "early life programming," which has an impact on future health outcomes.

In the first half of the $20^{\text {th }}$ century, based on epidemiological evidence, it has been demonstrated that adverse intrauterine environmental conditions may have consequences on adult health. The most tragic instance of prenatal epigenetic programming has been the Dutch famine during World War II. Scientists found out how genes could be literally reprogrammed based on intrauterine experience: the hunger suffered by mothers was associated in their offspring to an increased risk of cardiovascular disease and metabolic conditions like obesity, metabolic syndrome and adult diabetes. ${ }^{1}$

A recent review published by the Archives of Disease in Childhood on early life programming ${ }^{2}$ notes that, back in 1934, Kermack et al. analyzed historical mortality rates in England, Scotland and Sweden and referred that "figures acted as if the expectation of life was determined by the conditions existing in the years prior to the existence of the child". ${ }^{3}$ The authors maintained that improvements in childhood mortality largely depended on maternal health.

During the 1980s, Barker et al. suggested that a restricted fetal calorie intake results in a delayed fetal growth rate, reduces vital organ functioning and affects metabolic and endocrine mechanisms, making these infants more vulnerable to adverse environmental factors. ${ }^{4}$

This hypothesis assumes that, when facing an adverse environment during the intrauterine period and the first years of life, the body prepares a "predictive adaptive response" to an environment bereft of resources; however, in a postnatal environment with adequate resources, it poses a higher risk of noncommunicable diseases (NCDs) during adulthood (unexpected response). Such reasoning resulted in the concept of "Developmental Origins of Health and Disease", which indicates that exposure during early life may lead to epigenetic modifications (DNA methylation, histone modification, and small, non-coding RNA pieces) in programming, which have long-term effects caused by structural organ changes.

Several prenatal and postnatal environmental factors are described in the review as predisposing factors for NCDs. Maternal factors assumingly associated with a greater level of neurological disorders include infections, drug and alcohol use, certain medications, obesity and maternal stress, and prenatal overexposure to glucocorticoids and to environmental toxic agents such as arsenic and lead. Diet and weight gain, especially in the first year of life, are among postnatal factors to be considered and are related to an increased risk of cardiovascular disease (CVD), hypertension (HTN) and overweight. Preterm birth has been associated with metabolic disorders such as a reduced insulin sensitivity and higher levels of adiposity; also stress during infancy increases the risk of mental disorders and CVD.

Finally, it is worth noting the increasing evidence regarding exposure during early life and non-genomic transmission to future generations. ${ }^{5}$ Three cohort studies conducted in humans suggest potential instances of programmed effect transmission, two through the maternal line, ${ }^{6}$ and one through the paternal line. This study by Kaati et al. provides details of historical data on Swedish cohorts that demonstrated that food availability during grandparents' childhood had an impact on their grandchildren's risk of developing CVD and diabetes.?

Pregnancy and early childhood make up a critical period for the adequate growth and development of individuals. During this stage, the future is defined, not in terms of survival but of conditions and quality of life; the chances each child will have to grow and develop their potential as a healthy adult are being programmed. Knowing the consequences of "fetal programming" since the moment of conception provides a whole new dimension to the role of pediatricians.

Epidemiological studies have placed pediatrics in a privileged position to prevent NCDs by strengthening the concept of a fetal origin of adult disease. It is necessary for this concept, once known by neonatologists and dietitians, to become part of general pediatricians' armamentarium in current primary care settings 
so that they may offer timely recommendations and guidance to families regarding this stage of life aimed at preventing NCDs.

Disease prevention and health promotion actions should focus on the early detection of growth and development problems and exposure to environmental risks. A healthy diet and exercise during pregnancy, prolonged breastfeeding, a late complementary feeding introduction and protecting pregnant women against exposure to chemicals are some of the basic recommendations for this critical period.

Such measures require the joint responsibility and commitment of maternal and child health team members to ensure an adequate antenatal and perinatal care and an appropriate follow-up during early childhood, thus allowing individuals to be healthy throughout life.

Jorge Cabana M.D. President Sociedad Argentina de Pediatría http:/ /dx.doi.org/10.5546/aap.2016.eng.194

1. The Dutch Famine Birth Cohort Study. [Accessed on: January 12, 2016]. Available at: http:/ / www.dutchfamine. nl/index_files/study.htm

2. Williams TC, Drake AJ. What a general paediatrician needs to know about early life programming. Arch Dis Child 2015;100(11):1058-63.

3. Kermack WO, McKendrick AG, McKinlay PL. Death rates in Great Britain and Sweden some general regularities and their significance. Lancet 1934;223(5770):698-703.

4. Barker DJ, Osmond C. Infant mortality, childhood nutrition, and ischaemic heart disease in England and Wales. Lancet 1986;1(8489):1077-81.

5. Drake AJ, Liu L. Intergenerational transmission of programmed effects: public health consequences. Trends Endocrinol Metab 2010;21(4):206-13.

6. EmanuelI,FilaktiH, AlbermanE,EvansSJ.Intergenerational studies of human birthweight from the 1958 birth cohort. 1 . Evidence for a multigenerational effect. BrJObstet Gynaecol 1992;99(1):67-74.

7. Kaati G, Bygren LO, Edvinsson S. Cardiovascular and diabetes mortality determined by nutrition during parents' and grandparents' slow growth period. Eur J Hum Genet 2002;10(11):682-8. 